\title{
GLOBAL STABILITY \\ IN A NONAUTONOMOUS GENOTYPE SELECTION MODEL
}

BY

\author{
CHUANXI QIAN
}

Department of Mathematics and Statistics, Mississippi State University, Mississippi State, Mississippi

Abstract. Consider the nonautonomous difference equation

$$
y_{n+1}=\frac{y_{n} \exp \left(\beta_{n}\left(1-\sum_{i=0}^{k} \alpha_{i} y_{n-i}\right)\right)}{1-y_{n}+y_{n} \exp \left(\beta_{n}\left(1-\sum_{i=0}^{k} \alpha_{i} y_{n-i}\right)\right)}, \quad n=0,1, \ldots,
$$

where $k$ is a nonnegative integer, $\alpha_{0}, \alpha_{1}, \ldots, \alpha_{k-1}$ are nonnegative constants, $\alpha_{k}$ is a positive constant and $\left\{\beta_{n}\right\}$ is a nonnegative sequence, which is used as a genotype selection model. In this paper, we first establish some criteria for the positive equilibrium of Eq. (0.1) to be globally asymptotically stable. Then some special cases of Eq. (0.1) are investigated further and more global stability results are obtained. Our results also extend and improve some known results in the literature.

1. Introduction. Our aim in this paper is to study the global stability of the nonautonomous difference equation

$$
y_{n+1}=\frac{y_{n} \exp \left(\beta_{n}\left(1-\sum_{i=0}^{k} \alpha_{i} y_{n-i}\right)\right)}{1-y_{n}+y_{n} \exp \left(\beta_{n}\left(1-\sum_{i=0}^{k} \alpha_{i} y_{n-i}\right)\right)}, \quad n=0,1, \ldots,
$$

where

$$
\left\{\begin{array}{l}
k \in\{0,1, \ldots\}, \alpha_{0}, \alpha_{1}, \ldots, \alpha_{k-1} \in[0, \infty), \\
\alpha_{k} \in(0, \infty), \text { and }\left\{\beta_{n}\right\} \text { is a nonnegative sequence. }
\end{array}\right.
$$

When $k=0, \alpha_{0}=2$ and $\beta_{n} \equiv \beta$ (positive constant), Eq. (1.1) reduces to

$$
y_{n+1}=\frac{y_{n} \exp \left(\beta\left(1-2 y_{n}\right)\right)}{1-y_{n}+y_{n} \exp \left(\beta\left(1-2 y_{n}\right)\right)}, \quad n=0,1, \ldots,
$$

which was introduced by May [8] as an example of a map generated by a simple model for frequency-dependent natural selection. Eq. (1.3) gives the change in gene frequency between the $n$th generation and the next and the fitness function is $\exp (\beta(1-2 y))$. To

Received December 20, 2000.

2000 Mathematics Subject Classification. Primary 39A10, 92D25.

Key words and phrases. Nonautonomous difference equation, genotype selection model, globally asymptotic stability. 
increase the ecological realism. Grove et al. [1] introduced the delay in Eq. (1.3) and considered the following equation:

$$
y_{n+1}=\frac{y_{n} \exp \left(3\left(1-2 y_{n-k}\right)\right)}{1-y_{n}+y_{n} \exp \left(\beta\left(1-2 y_{n-k}\right)\right)}, \quad n=0,1 \ldots
$$

as a simple genotype selection model with one delay. The appearance of $y_{n-k}$ in the selection coefficient reflects the fact that the enviromment at the present time depends upon the activity of the population at some time in the past and that this in turn depends upon the gene frequency at that time. Then, in a later paper [3]. Grove et al. studied the genotype selection model with several delays:

$$
y_{n+1}=\frac{y_{n} \exp \left(\beta\left(1-\sum_{i=0}^{k} \alpha_{i} y_{n-i}\right)\right)}{1-y_{n}+y_{n} \exp \left(\beta\left(1-\sum_{i=0}^{k} \alpha_{i} y_{n-i}\right)\right)} . \quad n=0.1 \ldots
$$

The appearance of several delays in the model is incorporated with the phenomenon that fitness today depends on some function of the various life stages or ages today, which means the number of adults born $1.2 \ldots, k$ periods ago, each multiplied by the probability of surviving to the present and by the magnitude of their effect if they do not survive. For a discussion of the backgromel of this model in detail, see [3].

Asymptotic behavior of positive solutions of Eqs. (1.3) (1.5) has been studied by several authors. For example. the local stability of the positive equilibrium solution $\bar{y}=1 / 2$ of Eq. (1.3) was investigated by May in [8]; the oscillation and the stability and the periodic character of positive solutions of Eq. (1.4) were investigated in [1] and [2]: the oscillation and the stability of positive solutions of E(1. (1.5) were studied in [3]. Although some good results about the asymptotic behavior of positive solutions of Eqs. (1.3) (1.5) have been established, the discussion of the global stability of these equations is far from completed and this motivates us to further study this topic. By noting that the effects of a varying enviromment are important for evolutionary theory as the selective forces on systems in a fluctuating cnvironment differ from those in a stable environment and the assumptions of variation of the paraneters are a way of incorporating the variations in the enviromment, we assume that the parameter 3 in (1.5) varies with $n$ and so this leads us to study the nomautonomous equation (1.1).

Consider Eq. (1.1) with initial conditions $y_{-k}, y_{-k+1}, \ldots, y_{0} \in[0,1]$ and set $\alpha=$ $\sum_{i=0}^{k} \alpha_{i}$. When $\alpha \leq 1.0$ and 1 are only equilibrimm solutions, and when $\alpha>1.0 .1 / \alpha$ and 1 are the only equilibrium solutions of $\mathrm{Eq}$. (1.1). It is easy to see by induction that $y_{n} \in[0,1]$ for all $n \geq 0$. Also. if $y_{N}=0$ for some $N \geq 0$. then $y_{n}=0$ for all $n \geq N$, and if $y_{N}=1$ for some $N \geq 0$, then $y_{n}=1$ for all $n \geq N$. Hence, we will only consider the solutions $\left\{y_{n}\right\}$ of Eq. (1.1) with the following initial conditions:

$$
y_{-k}, y_{-k+1}, \ldots, y_{-1} \in[0.1] \text { and } y_{0} \in(0,1) \text {. }
$$

Then, clearly, $y_{n} \in(0,1)$ for all $n \geq 0$. In the following sections, we will always assume that $a>1$. We will establish a sufficient condition such that $\bar{y}=1 / a$ is globally asymptotically stable in the sense that $\bar{y}$ is stable and attracts all positive solutions with initial conditions of the form (1.6). We will also further discuss the globally asymptotic stability of some special cases of $\mathrm{E}_{1}$. (1.1). Our results will extend and improve some results established in [1] and [3]. 
2. Global stability of Equation (1.1). In this section, we study the global stability of Eq. (1.1). By introducing the substitution

$$
x_{n}=\frac{y_{n}}{1-y_{n}} \quad \text { for } 0<y_{n}<1, n=-k,-k+1, \ldots,
$$

Eq. (1.1) with initial conditions (1.6) becomes

$$
x_{n+1}=x_{n} \exp \left[\beta_{n}\left(1-\sum_{i=0}^{k} \alpha_{i} \frac{x_{n-i}}{1+x_{n-i}}\right)\right], \quad n=0,1, \ldots,
$$

with initial conditions

$$
x_{-k}, x_{-k+1}, \ldots, x_{-1} \in[0, \infty) \text { and } x_{0} \in(0, \infty) .
$$

Clearly, $\bar{y}$ is stable and attracts all positive solutions of Eq. (1.1) with initial conditions of the form (1.6) if and only if the equilibrium $\bar{x}=\frac{1}{\alpha-1}$ of Eq. (2.2) is stable and attracts all positive solutions of Eq. (2.2) with initial conditions of the form (2.3).

To establish our global stability result, we need the following two lemmas.

Lemma $1([9])$. Consider the difference equation

$$
x_{n+1}=x_{n} h\left(n, x_{n}, x_{n-1}, \ldots, x_{n-k}\right), \quad n=0,1, \ldots,
$$

where for each $n, h \in C\left[[0, \infty)^{k+1},(0, \infty)\right]$ and $h$ is nonincreasing in each of its arguments. Assume that $\bar{x}$ is the unique positive equilibrium of Eq. (2.4) and that

$$
\prod_{n=0}^{\infty} h(n, x, x, \ldots, x)=\infty \text { for } 0<x<\bar{x}
$$

and

$$
\prod_{n=0}^{\infty} h(n, x, x, \ldots, x)=0 \text { for } \bar{x}<x<\infty .
$$

Suppose also that there exists a nonincreasing function $H \in C[[0, \infty),(0, \infty)]$ such that for $n \geq 0$,

$$
\prod_{j=n}^{n+k} h(n, x, x, \ldots, x) \leq H(x) \quad \text { for } 0<x<\bar{x}
$$

and

$$
\prod_{j=n}^{n+k} h(n, x, x, \ldots, x) \geq H(x) \text { for } \bar{x}<x<\infty .
$$

Finally, assume that the solution of the equation

$$
z_{n+1}=\bar{x} H\left(z_{n}\right), \quad n=0,1, \ldots,
$$

with $z_{0}=\bar{x} H(0)$ tends to $\bar{x}$ as $n$ tends to $\infty$. Then every positive solution of Eq. (2.4) tends to $\bar{x}$ as $n$ tends to $\infty$.

LEMma 2 ([4]). Consider the difference equation

$$
w_{n+1}=\exp \left(a \frac{1-w_{n}}{1+b w_{n}}\right), \quad n=0,1, \ldots,
$$


where $a \in(0, \infty), b \in[0, \infty)$, and $u_{0} \in[0, \infty)$. If

$$
\frac{a}{1+b} \leq 1
$$

then the positive equilibrium $\bar{w}=1$ is a global attractor of all positive solutions of $(2.10)$.

The following theorem is our main result in this section.

TheOrem 1. Assume that (1.2) holds and that

$$
\sum_{n=0}^{\infty} \beta_{n}=\infty \quad \text { and } \quad \sum_{j=n}^{n+k} \beta_{j} \leq \frac{\alpha}{\alpha-1} \quad \text { for } n \geq 0 .
$$

Then the positive equilibrium $\bar{y}=1 / \alpha$ of Eq. (1.1) is globally asymptotically stable.

Proof. From the above discussion, we see that it suffices to show that the equilibrium solution $\bar{x}=\frac{1}{\alpha-1}$ of Eq. (2.2) is stable and attracts all positive solutions of Eq. (2.2). First, we show that $\bar{x}$ is a global attractor of all positive solutions. Let

$$
h\left(n, u_{0}, u_{1} \ldots u_{k}\right)=\exp \left[\beta_{n}\left(1-\sum_{i=0}^{k} \alpha_{i} \frac{u_{i}}{1+u_{i}}\right)\right] .
$$

Clearly, for each $n, h \in C\left[[0, \infty)^{k+1},(0, \infty)\right], h$ is nonincreasing in each of its arguments and $h$ satisfies $(2.5)$ and $(2.6)$ with $\bar{x}=\frac{1}{\alpha-1}$. Let

$$
B=\sup \left\{\sum_{j=n}^{n+k} \beta_{j}: n=0,1, \ldots\right\} \text {. }
$$

Observe that

$$
\begin{aligned}
\prod_{j=n}^{n+k} h(j, x, \ldots . x) & =\prod_{j=n}^{n+k} \exp \left(\beta_{j}\left(1-\sum_{i=0}^{k} \alpha_{i} \frac{x}{1+x}\right)\right) \\
& =\prod_{j=n}^{n+k} \exp \left(\beta_{j}\left(1-\alpha \frac{x}{1+x}\right)\right) \\
& =\exp \left(\sum_{j=n}^{n+k} \beta_{j}\left(1-\alpha \frac{x}{1+x}\right)\right) \\
& \leq \exp \left(B\left(1-\alpha \frac{x}{1+x}\right)\right) \text { for } 0<x<\bar{x}
\end{aligned}
$$

and similarly,

$$
\prod_{j=n}^{n+k} h(j, x, \ldots, x) \geq \exp \left(B\left(1-\alpha \frac{x}{1+x}\right)\right) \quad \text { for } \bar{x}<x<\infty .
$$

Let

$$
H(x)=\exp \left(B\left(1-\alpha \frac{x}{1+x}\right)\right), \quad x \geq 0 .
$$


Then (2.7) and (2.8) hold and so by Lemma 1, it suffices to show that every positive solution of the equation

$$
z_{n+1}=\frac{1}{\alpha-1} \exp \left(B\left(1-\alpha \frac{z_{n}}{1+z_{n}}\right)\right)
$$

tends to its positive equilibrium $\bar{z}=\bar{x}=\frac{1}{\alpha-1}$. To this end, let $w_{n}=(\alpha-1) z_{n}$. Then every positive solution of Eq. (2.13) tends to $\bar{z}$ if and only if every positive solution of the equation

$$
w_{n+1}=\exp \left(B \frac{1-w_{n}}{1+\frac{1}{\alpha-1} w_{n}}\right)
$$

tends to the equilibrium $\bar{w}=1$. By Lemma 2 , we see that $\bar{w}$ is a global attractor of all positive solutions of Eq. (2.14) if $\frac{B}{1+\frac{1}{\alpha-1}} \leq 1$, which is equivalent to (2.12). Hence, $\bar{z}$ is a global attractor of Eq. (2.13) and so $\bar{y}$ is a global attractor of Eq. (1.1).

Next, we show that the equilibrium solution $\bar{x}=\frac{1}{\alpha-1}$ of Eq. (2.2) is stable. To this end, consider the sequence $\left\{z_{n}\right\}$ defined by (2.13) and $z_{0}=\frac{1}{\alpha-1} \exp (B)$. From the above discussion, we know that $z_{n} \rightarrow \bar{x}$ as $n \rightarrow \infty$. Hence, for any $\varepsilon>0$, there exists an $N>0$ such that

$$
\left|z_{n}-\bar{x}\right|<\varepsilon \text { for all } n \geq 2 N \text {. }
$$

In addition, from the definition of $\left\{z_{n}\right\}$, it is easy to see that

$$
z_{2 n+1}<z_{2(n+1)+1}<\bar{x}<z_{2(n+1)}<z_{2 n} \text { for } n \geq 0 .
$$

Then by the continuous dependence of solutions on the initial conditions, there exists a positive number $\delta$ such that if $\left\{x_{n}\right\}$ is a solution of Eq. (2.2) with initial conditions satisfying

$$
\left|x_{n}-\bar{x}\right|<\delta, \quad n=-k,-k+1, \ldots, 0,
$$

then

$$
z_{2 N+1} \leq x_{n} \leq z_{2 N}, \quad n=0,1, \ldots, 2 N+1
$$

We claim that

$$
z_{2 N+1} \leq x_{n} \leq z_{2 N}, \quad n \geq 2 N+2
$$

First we show that

$$
z_{2 N+1} \leq x_{2 N+2} \leq z_{2 N}, \quad n=0,1, \ldots, 2 N+1 .
$$

We assume that $x_{2 N+2} \leq x_{2 N+1}$; the proof for the case that $x_{2 N+2} \geq x_{2 N+1}$ is similar and will be omitted. Clearly, if $x_{2 N+2} \geq \bar{x}$, then $x_{2 N+2}$ satisfies (2.20). Now, suppose that $x_{2 N+2}<\bar{x}$. Let $x_{n}=\bar{x} e^{w_{n}}$. Then $\left\{w_{n}\right\}$ satisfies the equation

$$
w_{n+1}-w_{n}+\frac{(\alpha-1) \beta_{n}}{\alpha} \sum_{i=0}^{k} \alpha_{i} \frac{e^{w_{n-i}}-1}{\alpha-1+e^{w_{n-i}}}=0 .
$$

By noting that $x_{2 N+2}<\bar{x}$ and $x_{2 N+2} \leq x_{2 N+1}$, we see that

$$
w_{2 N+2} \leq 0 \quad \text { and } \quad w_{2 N+2} \leq w_{2 N+1} .
$$


Then it follows from (2.21) and (2.22) that

$$
\frac{(\alpha-1) \beta_{2 N+1}}{\alpha} \sum_{i=0}^{k} \alpha_{i} \frac{1-e^{w_{2 N+1-i}}}{\alpha-1+e^{w_{2 N+1-i}}}=w_{2 N+2}-w_{2 N+1} \leq 0,
$$

which implies that there exists an $m$ with $0 \leq m \leq k$ such that $w_{2 N+1-m} \geq 0$. Hence.

$$
w_{2 N+2} \geq w_{2 N+2}-w_{2 N+1-m}=\sum_{j=2 N+1-m}^{2 N+1} \frac{(\alpha-1) \beta_{j}}{\alpha} \sum_{i=0}^{k} \alpha_{i} \frac{1-e^{w_{j-i}}}{\alpha-1+e^{w_{j-i}}} .
$$

Then by noting $e^{w_{n}}=(\alpha-1) x_{n}$ and (2.18), we see that

$$
e^{w_{i}} \leq(\alpha-1) z_{2 N}, \quad i=0,1, \ldots, 2 N+1 .
$$

Hence, it follows from (2.23) that

$$
\begin{aligned}
w_{2 N+2} & \geq \sum_{j=2 N+1-m}^{2 N+1} \frac{(\alpha-1) \beta_{j}}{\alpha} \sum_{i=0}^{k} \alpha_{i} \frac{1-(\alpha-1) z_{2 N}}{\alpha-1+(\alpha-1) z_{2 N}} \\
& \geq \sum_{j=2 N+1-k}^{2 N+1} \frac{(\alpha-1) \beta_{j}}{\alpha} \sum_{i=0}^{k} \alpha_{i} \frac{1-(\alpha-1) z_{2 N}}{\alpha-1+(\alpha-1) z_{2 N}} \\
& =\sum_{j=2 N+1-k}^{2 N+1} \frac{(\alpha-1) \beta_{j}}{\alpha} \sum_{i=0}^{k} \alpha_{i} \frac{1-(\alpha-1) z_{2 N}}{\alpha-1+(\alpha-1) z_{2 N}} \\
& \geq B\left(1-\alpha \frac{z_{2 N}}{1+z_{2 N}}\right)
\end{aligned}
$$

which implies that

$$
(\alpha-1) x_{2 N+2} \geq \exp \left(B\left(1-\alpha \frac{z_{2 N}}{1+z_{2 N}}\right)\right)
$$

Hence,

$$
x_{2 N+2} \geq \frac{1}{\alpha-1} \exp \left(B\left(1-\alpha \frac{z_{2 N}}{1+z_{2 N}}\right)\right) \geq z_{2 N+1} .
$$

Then by noting $x_{2 N+2} \leq x_{2 N+1}$ and $x_{2 N+1} \leq z_{2 N}$, we see that $(2.20)$ holds.

An easy induction and an argument similar to the one above, shows that (2.19) holds. Finally, by noting (2.15), (2.16), (2.18), and (2.19), we see that

$$
\left|x_{n}-\bar{x}\right|<\varepsilon \text { for all } n \geq 2 N
$$

and so by the definition of stability, $\bar{x}$ is stable. The proof is complete.

REMARK 1. When $\beta_{n} \equiv \beta$ is a positive constant, Eq. (1.1) reduces to the autonomous equation (1.5), and the condition (2.12) for the globally asymptotical stability becomes

$$
\beta \leq \frac{\alpha}{(k+1)(\alpha-1)}
$$

It has been shown in [3] that the equilibrium $\bar{y}=1 / \alpha$ of $\mathrm{Eq}_{1}$. (1.5) is locally stable if

$$
\alpha_{0} \neq 0, \quad \alpha<2 \alpha_{0}, \quad \beta<\frac{2 \alpha}{\alpha-1} ;
$$


or

$$
\alpha_{0}=0, \quad \beta<\frac{\alpha^{2}}{(\alpha-1) \sum_{i=1}^{k} \alpha_{i} i}
$$

and $\bar{y}=1 / \alpha$ is a global attractor of positive solutions if

$$
k \geq 1 \quad \text { and } \beta \leq \min \left\{\frac{4}{\alpha_{k}+\alpha_{0}}, \frac{\alpha^{2}}{k(\alpha-1)\left(\alpha-\alpha_{0}\right)}\right\} .
$$

Here, our condition (2.24) is not only different from the above conditions established in [3], but also a sufficient condition for the equilibrium solution $\bar{y}=1 / \alpha$ of Eq. (1.5) to be globally asymptotically stable.

3. Global stability of Equation (1.1) with one delay and constant coefficients. In this section, we further study the globally asymptotical stability of the autonomous difference equation

$$
y_{n+1}=\frac{y_{n} e^{\beta\left(1-\alpha y_{n-k}\right)}}{1-y_{n}+y_{n} e^{\beta\left(1-\alpha y_{n-k}\right)}},
$$

which is a special case of Eq. (1.1) with $k \geq 1, \alpha_{0}=\alpha_{1}=\cdots=\alpha_{k-1}=0, \alpha_{k}=\alpha$, and $\beta_{n} \equiv \beta$, a positive constant.

We need the following lemma in the proof of our result.

Lemma 3 ([7]). Consider the difference equation

$$
x_{n+1}-x_{n}+p_{n} f\left(x_{n-k}\right)=0, \quad n=0,1, \ldots,
$$

where $\left\{p_{n}\right\}$ is a nonnegative sequence and $f \in C[R, R]$ with $x f(x)>0$ if $x \neq 0$. Assume that

$$
|f(x)|<|x| \quad \text { if } x \neq 0
$$

and

$$
\sum_{n=0}^{\infty} p_{i}=\infty \quad \text { and } \quad \sum_{i=n-k}^{n} p_{i} \leq \frac{3}{2}+\frac{1}{2(k+1)}
$$

for all large $n$. Then every solution of Eq. (3.2) tends to zero as $n \rightarrow \infty$.

The following theorem is our main result in this section.

Theorem 2. Consider Eq. (3.1) and assume that

$$
\beta \leq \frac{4}{\alpha(k+1)}\left(\frac{3}{2}+\frac{1}{2(k+1)}\right) .
$$

Then the positive equilibrium solution $\bar{y}=1 / \alpha$ is globally asymptotically stable.

Proof. We first show that $\bar{y}$ is a global attractor of positive solutions of Eq. (3.1). By using substitution (2.1), Eq. (3.1) becomes

$$
x_{n+1}=x_{n} \exp \left(\beta\left(1-\alpha \frac{x_{n-k}}{1+x_{n-k}}\right)\right), \quad n=0,1, \ldots
$$


Let $x_{n}=\frac{1}{\alpha-1} e^{z_{n}}$. Then Eq. (3.6) reduces to

$$
z_{n+1}-z_{n}+\left(\frac{\alpha \beta}{4}\right) \frac{4(\alpha-1)}{\alpha} \frac{e^{z_{n-k}}-1}{\alpha-1+e^{z_{n-k}}}=0,
$$

which is in the form (3.2) with

$$
f(x)=\frac{4(\alpha-1)}{\alpha} \frac{e^{x}-1}{\alpha-1+e^{x}} \text { and } \quad p_{n} \equiv \frac{\alpha \beta}{4} .
$$

To show that $\bar{y}$ is a global attractor, it suffices to show that every solution of Eq. (3.7) tends to zero. Observing that $x f(x)>0$ for $x \neq 0$ and

$$
(x-f(x))^{\prime}=\frac{\left(\alpha-1-e^{x}\right)^{2}}{\left(\alpha-1+e^{x}\right)^{2}}>0 \quad \text { for } x \neq \ln (\alpha-1),
$$

we see that

$$
|f(x)|<|x| \text { for } x \neq 0
$$

and so (3.3) holds. Then by noting (3.5), we find that (3.4) holds also. Hence, all the conditions assumed in Lemma 3 are satisfied and so every solution of Eq. (3.1) tends to zero. Then it follows that $\bar{y}$ is a global attractor.

Next, we show that $\bar{y}$ is stable also. Clearly, it suffices to show that the zero solution of Eq. (3.7) is stable. To this end, observe that the linearized equation of (3.7) is

$$
w_{n+1}-w_{n}+\frac{\alpha-1}{\alpha} \beta w_{n-k}=0 .
$$

It is well known that (see [5], for example) the zero solution of the linear equation (3.8) is asymptotically stable if and only if

$$
\beta<\frac{2 \alpha}{\alpha-1} \cos \frac{k \pi}{2 k+1} .
$$

Hence, by linearized stability theory, if (3.9) holds, then the trivial solution of Eq. (3.7) is locally asymptotically stable. Therefore, to complete the proof, we only need to show that for any $k \geq 1$,

$$
\frac{2 \alpha}{\alpha-1} \cos \frac{k \pi}{2 k+1}>\frac{4}{\alpha(k+1)}\left[\frac{3}{2}+\frac{1}{2(k+1)}\right],
$$

that is,

$$
\frac{\alpha^{2}}{4(\alpha-1)} \cos \frac{k \pi}{2 k+1}>\frac{1}{2(k+1)}\left[\frac{3}{2}+\frac{1}{2(k+1)}\right] .
$$

To this end, first observe that

$$
\inf \left\{\frac{\alpha^{2}}{4(\alpha-1)}: \alpha>1\right\}=1 .
$$

Hence, it would be sufficient to show that the inequality

$$
\cos \frac{\pi k}{2 k+1} \geq \frac{1}{2(k+1)}\left[\frac{3}{2}+\frac{1}{2(k+1)}\right]
$$

holds for any $k \geq 1$. By noting

$$
\cos \frac{\pi}{3}>\frac{7}{16}=\frac{1}{2(1+1)}\left[\frac{3}{2}+\frac{1}{2(1+1)}\right]
$$


and

$$
\cos \frac{2 \pi}{5}>\frac{5}{18}=\frac{1}{2(2+1)}\left[\frac{3}{2}+\frac{1}{2(2+1)}\right]
$$

we see that $(3.10)$ holds when $k=1$ and $k=2$, and so it suffices to show that the following function inequality holds:

$$
\cos \frac{\pi x}{2 x+1}>\frac{1}{2(x+1)}\left[\frac{3}{2}+\frac{1}{2(x+1)}\right], \quad x \geq 3 .
$$

Let $t=\frac{\pi x}{2 x+1}$. Then (3.11) becomes

$$
\cos t>\frac{\pi-2 t}{2(\pi-t)}\left[\frac{3}{2}+\frac{1}{2}\left(\frac{\pi-2 t}{\pi-t}\right)\right], \quad \frac{3 \pi}{7} \leq t<\frac{\pi}{2},
$$

which can be rearranged in the form

$$
\cos t>\frac{4 \pi^{2}-13 \pi t+10 t^{2}}{4(\pi-t)^{2}}, \quad \frac{3 \pi}{7} \leq t<\frac{\pi}{2} .
$$

Now, consider the functions

$$
g(t)=\cos t \quad \text { and } \quad h(t)=\frac{4 \pi^{2}-13 \pi t+10 t^{2}}{4(\pi-t)^{2}}, \quad \frac{3 \pi}{7} \leq t \leq \frac{\pi}{2} .
$$

Observe that

$$
g^{\prime \prime}(t)=-\cos t<0 \quad \text { and } \quad h^{\prime \prime}(t)=\frac{7 \pi t-4 \pi^{2}}{2(\pi-t)^{4}}<0, \quad \frac{3 \pi}{7}<t<\frac{\pi}{2} .
$$

We see that

$$
g^{\prime}(t)<g^{\prime}\left(\frac{3 \pi}{7}\right)=-\sin \frac{3 \pi}{7} \quad \text { and } \quad h^{\prime}(t)>h^{\prime}\left(\frac{\pi}{2}\right)=-\frac{3}{\pi} \quad \text { for } \quad \frac{3 \pi}{7}<t<\frac{\pi}{2} .
$$

Since $\sin 3 \pi / 7>3 / \pi$, it follows that $g^{\prime}(x)<h^{\prime}(x)$ on the interval $(3 \pi / 7, \pi / 2)$. Then by noting $g(\pi / 2)=h(\pi / 2)=0$ also, we see that $g(t)>h(t)$ for $3 \pi / 7 \leq t<\pi / 2$, that is, (3.11) holds. The proof is complete.

REMARK 2. (1) By noting that Lemma 3 is about nonautonomous equations and by using the same argument used in the proof of the above theorem, we may have the following global stability result for the nonautonomous difference equation:

$$
y_{n+1}=\frac{y_{n} e^{\beta_{n}\left(1-\alpha y_{n-k}\right)}}{1-y_{n}+y_{n} e^{\beta_{n}\left(1-\alpha y_{n-k}\right)}} .
$$

If $\sum_{n=0}^{\infty} \beta_{n}=\infty$ and for all large $n$,

$$
\sum_{i=n-k}^{n} \beta_{i} \leq \frac{4}{\alpha}\left[\frac{3}{2}+\frac{1}{2(k+1)}\right],
$$

then $\bar{y}=1 / \alpha$ is a global attractor of all positive solutions of Eq. (3.12).

(2) When $\alpha=2$, Condition (3.5) reduces to

$$
\beta \leq \frac{2}{k+1}\left[\frac{3}{2}+\frac{1}{2(k+1)}\right] .
$$

For this special case of Eq. (3.1), it has been shown in [1] that the equilibrium solution $\bar{y}=1 / 2$ is globally asymptotically stable if one of the following conditions holds:

(a) $k=1$ and $0<\beta \leq 2$; 
(b) $k \geq 2$ and $0<\beta \leq 2 / k$.

Clearly, for the case that $k \geq 2$, our condition (3.13) is better than the above condition established in [1].

(3) Although we believe that (3.9) is a necessary and sufficient condition for the global stability of the equilibrium solution $\bar{y}=1 / \alpha$ of Eq. (3.1), we cannot prove it at this moment. However, the gap between condition (3.5) and (3.9) is not very big when $\alpha$ is not very close to 1 and $\alpha$ is not very large. For instance, when $\alpha=2$ and $k=3$,

$$
\frac{2 \alpha}{\alpha-1} \cos \frac{k \pi}{2 k+1}=4 \cos \frac{3 \pi}{7} \approx 0.89
$$

while

$$
\frac{4}{\alpha(k+1)}\left[\frac{3}{2}+\frac{1}{2(k+1)}\right]=\frac{2}{4}\left[\frac{3}{2}+\frac{1}{8}\right] \approx 0.81 .
$$

4. Global stability of Equation (1.1) without delay. In this section, we further study the global asymptotical stability of the special case of Eq. (1.1) without delay:

$$
y_{n+1}=\frac{y_{n} e^{\beta_{n}\left(1-\alpha y_{n}\right)}}{1-y_{n}+y_{n} e^{\beta_{n}\left(1-\alpha y_{n}\right)}} .
$$

We need the following result, which is extracted from [6].

Lemma $4([6])$. Consider the difference equation

$$
x_{n+1}=f\left(n, x_{n}\right), \quad n=0,1, \ldots
$$

where $f:\{0,1, \ldots\} \times[0, \infty) \rightarrow[0, \infty), f(n, x)$ is continuous in $x$ and $f(n, \bar{x}) \equiv \bar{x}$ where $\bar{x}$ is a positive constant. If there exists a positive definite and decrescent function $V(n, x)$ with respect to $\bar{x}$ such that

$$
\Delta V\left(n, x_{n}\right)=V\left(n+1, f\left(n, x_{n}\right)\right)-V\left(n, x_{n}\right) \leq 0, \quad n=0,1, \ldots,
$$

then $\bar{x}$ is stable; furthermore, for any solution $\left\{x_{n}\right\}$ of Eq. (4.2) if there is a continuous function $\omega(x) \geq 0$ defined for $x \geq 0$ such that

$$
\Delta V\left(n, x_{n}\right) \leq-\omega\left(x_{n}\right), \quad n=0,1, \ldots,
$$

then either $\left\{x_{n}\right\}$ is unbounded or it approaches the set

$$
E=\{x \in[0, \infty): \omega(x)=0\}
$$

The following theorem is our main result in this section.

Theorem 3. Assume that there exist positive constants $b$ and $\beta$ such that $b \leq \beta_{n} \leq \beta$ for $n \geq 0$, and

$$
\left(1-\frac{4}{\alpha \beta}\right) e^{\beta-2} \leq 1 .
$$

Then the equilibrium solution $\bar{y}=1 / \alpha$ is globally asymptotically stable. 
Proof. By using the substitution (2.1), Eq. (4.1) becomes

$$
x_{n+1}=x_{n} \exp \left(\beta_{n} \frac{1-(\alpha-1) x_{n}}{1+x_{n}}\right),
$$

and it suffices to show that the equilibrium solution $\bar{x}=\frac{1}{\alpha-1}$ of Eq. (4.4) is globally asymptotically stable. To this end, let

$$
V(n, x)=(x-\bar{x})^{2} \quad \text { for } n \geq 0 \text { and } x \geq 0 .
$$

Then

$$
\begin{aligned}
\Delta V\left(n, x_{n}\right)= & x_{n}\left[\exp \left(\beta_{n} \frac{1-(\alpha-1) x_{n}}{1+x_{n}}\right)-1\right] \\
& \times\left[x_{n} \exp \left(\beta_{n} \frac{1-(\alpha-1) x_{n}}{1+x_{n}}\right)+x_{n}-2 \bar{x}\right] .
\end{aligned}
$$

By noting that the function $\exp \left(\beta_{n} \frac{1-(\alpha-1) x}{1+x}\right)-1$ is decreasing with respect to $x$ and is zero at $\bar{x}$, it is easy to see that

$$
\begin{aligned}
& \left(x_{n}-\bar{x}\right)\left[\exp \left(\beta_{n} \frac{1-(\alpha-1) x_{n}}{1+x_{n}}\right)-1\right] \\
& \leq\left(x_{n}-\bar{x}\right)\left[\exp \left(b \frac{1-(\alpha-1) x_{n}}{1+x_{n}}\right)-1\right]<0 \quad \text { for } x_{n} \neq \bar{x} .
\end{aligned}
$$

We claim that

$$
\begin{aligned}
& \left(x_{n}-\bar{x}\right)\left[x_{n} \exp \left(\beta_{n} \frac{1-(\alpha-1) x_{n}}{1+x_{n}}\right)+x_{n}-2 \bar{x}\right] \\
& \quad \geq\left(x_{n}-\bar{x}\right)\left[x_{n} \exp \left(\beta \frac{1-(\alpha-1) x_{n}}{1+x_{n}}\right)+x_{n}-2 \bar{x}\right]>0 \quad \text { for } x_{n} \neq \bar{x} .
\end{aligned}
$$

First, observe that

$$
\begin{aligned}
x_{n} \exp \left(\beta \frac{1-(\alpha-1) x_{n}}{1+x_{n}}\right)+x_{n} & -2 \bar{x} \\
& \geq x_{n} \exp \left(\beta_{n} \frac{1-(\alpha-1) x_{n}}{1+x_{n}}\right)+x_{n}-2 \bar{x} \text { for } x_{n}<\bar{x}
\end{aligned}
$$

and

$$
\begin{aligned}
& x_{n} \exp \left(\beta \frac{1-(\alpha-1) x_{n}}{1+x_{n}}\right)+x_{n}-2 \bar{x} \\
& \quad \leq x_{n} \exp \left(\beta_{n} \frac{1-(\alpha-1) x_{n}}{1+x_{n}}\right)+x_{n}-2 \bar{x} \text { for } x_{n}>\bar{x} .
\end{aligned}
$$

Hence, to show that (4.7) holds, it suffices to show that

$$
\left(x_{n}-\bar{x}\right)\left[x_{n} \exp \left(\beta \frac{1-(\alpha-1) x_{n}}{1+x_{n}}\right)+x_{n}-2 \bar{x}\right] \geq 0 \quad \text { for } x_{n} \neq \bar{x}
$$

To this end, let

$$
h(x)=x \exp \left(\beta \frac{1-(\alpha-1) x}{1+x}\right)+x-2 \bar{x} \text { for } x \geq 0 .
$$


Observe that

$$
h^{\prime}(x)=\exp \left(\beta \frac{1-(\alpha-1) \cdot x}{1+x}\right)\left[1-\frac{\alpha \beta x}{(1+x)^{2}}\right]+1
$$

and

$$
h^{\prime \prime}(x)=\exp \left(\beta \frac{1-(\alpha-1) x}{1+x}\right)\left[\frac{\alpha \beta((\alpha \beta-2) x-2)}{(1+x)^{4}}\right] .
$$

If $\alpha \beta \leq 2$, then $h^{\prime \prime}(x)<0$ and so $h^{\prime}(x)>h^{\prime}(\infty)=e^{-(\alpha-1) \beta}+1>0$; if $\alpha \beta>2$, then

$$
h^{\prime \prime}(x)>0 \quad \text { for } x>\frac{1}{\alpha \beta-2} \quad \text { and } \quad h^{\prime \prime}(x)<0 \quad \text { for } x<\frac{1}{\alpha \beta-2}
$$

and so

$$
h^{\prime}(x)>h^{\prime}\left(\frac{1}{\alpha \beta-2}\right)=\left(\frac{4}{\alpha \beta}-1\right) \exp (\beta-2)+1 \quad \text { for } x \neq \frac{1}{\alpha \beta-2} .
$$

which, in view of (4.3), yields $h^{\prime}(x)>0$ for $x \neq \frac{1}{\alpha \beta-2}$. Then, by noting $h(\bar{x})=0$ also, we see that $(x-\bar{x}) h(x)>0$ for $x \neq \bar{x}$, which implies that (4.8) holds. Hence, (4.7) is true. Finally, by noting (4.6) and (4.7), it follows from (4.5) that

$$
\begin{aligned}
\Delta V\left(n, x_{n}\right)= & \frac{x_{n}}{\left(x_{n}-\bar{x}\right)^{2}}\left(x_{n}-\bar{x}\right)\left[\exp \left(\beta_{n} \frac{1-(\alpha-1) x_{n}}{1+x_{n}}\right)-1\right] \\
& \times\left(x_{n}-\bar{x}\right)\left[x_{n} \exp \left(\beta_{n} \frac{1-(\alpha-1) x_{n}}{1+x_{n}}\right)+x_{n}-2 \bar{x}\right] \\
\leq & \frac{x_{n}}{\left(x_{n}-\bar{x}\right)^{2}}\left(x_{n}-\bar{x}\right)\left[\exp \left(b \frac{1-(\alpha-1) x_{n}}{1+x_{n}}\right)-1\right] \\
& \times\left(x_{n}-\bar{x}\right)\left[x_{n} \exp \left(\beta \frac{1-(\alpha-1) x_{n}}{1+x_{n}}\right)+x_{n}-2 \bar{x}\right] \\
< & 0 \text { for } x_{n} \neq \bar{x} .
\end{aligned}
$$

Let

$$
\omega(x)=-x\left[\exp \left(b \frac{1-(\alpha-1) x}{1+x}\right)-1\right]\left[x \exp \left(\beta \frac{1-(\alpha-1) x}{1+x}\right)+x-2 \bar{x}\right] .
$$

From (4.9) we see that

$$
\Delta V\left(n, x_{n}\right) \leq-\omega\left(x_{n}\right) \leq 0
$$

and

$$
E=\{x \in[0, \infty), \omega(x)=0\}=\{\bar{x}\} .
$$

Also, $\Delta\left(n, x_{n}\right) \leq 0$ yields

$$
\left|x_{n+1}-\bar{x}\right| \leq\left|x_{n}-\bar{x}\right|, \quad n=0,1, \ldots,
$$

which clearly implies that every solution $\left\{x_{n}\right\}$ of Eq. (4.4) is bounded. Therefore, by Lemma 4, the equilibrium solution $\bar{x}=\frac{1}{\alpha-1}$ of $\mathrm{Eq}$. (4.4) is stable and every positive solution of the equation tends to $\bar{x}$, that is, $\bar{x}$ is globally asymptotically stable. Then, it follows that the equilibrium solution $\bar{y}=\frac{1}{\alpha}$ of Eq. (4.1) is globally asymptotically stable. The proof is complete. 
REMARK 3. When $\beta_{n} \equiv \beta$ is a positive constant, Eq. (4.1) reduces to the autonomous difference equation

$$
y_{n+1}=\frac{y_{n} e^{\beta\left(1-\alpha y_{n}\right)}}{1-y_{n}+y_{n} e^{\beta\left(1-\alpha y_{n}\right)}} .
$$

It has been shown in [3] that if $\alpha>1, \alpha \neq 2$ and

$$
\beta \leq \frac{8}{\alpha},
$$

then the equilibrium $\bar{y}=1 / \alpha$ of Eq. (4.10) is globally asymptotically stable. From our condition (4.3) we see that $\beta \leq 2$ is a sufficient condition for $\bar{y}$ to be globally asymptotically stable. While for the case that $\beta>2,(4.3)$ is equivalent to

$$
\alpha \leq \frac{4}{\beta\left(1-e^{2-\beta}\right)} .
$$

It is easy to check that if $\beta \leq 2+\ln 2$, then $\frac{4}{\beta\left(1-e^{2-\beta}\right)}>\frac{8}{\beta}$ and so (4.3) is better than (4.11) in this case.

\section{REFERENCES}

[1] E. A. Grove, V. L. Kocić, G. Ladas, and R. Levins, Oscillation and stability in a simple genotype selection model, Quart. Appl. Math. 52 , 499-508 (1994)

[2] E. A. Grove, V. L. Kocić and R. Levins, Periodicity in a simple genotype selection model, Differential Equations Dynam. Systems 1, 35-50 (1993)

[3] E. A. Grove, V. L. Kocić, G. Ladas, and R. Levins, Oscillation and stability in a genotype selection model and several delays. Difference Equations: theory and applications, J. Differential Equations Appl. 2, 205-217 (1996)

[4] E. A. Grove, G. Ladas, and C. Qian, Global attractivity in a "food-limited" population model, Dynamical Systems Appl. 2, 243-250 (1993)

[5] V. L. Kocić and G. Ladas, Global Asymptotic Behavior of Nonlinear Difference Equations of Higher Order with Applications, Kluwer Academic Publishers, Dordrecht, 1993

[6] V. Lakshmikantham and D. Trigiante, Theory of Difference Equations: Numerical Methods and Applications, Academic Press, Inc., 1987

[7] H. Matsunaga, T. Hara, and S. Sakata, Global attractivity for a nonlinear difference equation with variable delay, Comput. Math. Appl. 41, 543-551 (2001)

[8] R. M. May, Course 8: Nonlinear problems in ecology and resource management, in R. H. G. Helleman, G. Iooss, and R. Stora, editors, Chaotic Behavior of Deterministic Systems, NorthHolland Publ. Co., 1983

[9] C. Qian, Global attractivity in nonlinear delay difference equations, Bull. Institute Math. Acad. Sinica 24, 187-204 (1996) 